\title{
A brief report of toxicity end points of HER2 vaccines for the treatment of patients with HER2 ${ }^{+}$ breast cancer
}

This article was published in the following Dove Medical Press journal: Drug Design, Development and Therapy

\author{
Ricardo Costa' \\ Saif Zaman ${ }^{2}$ \\ Susan Sharpe ${ }^{3}$ \\ Irene Helenowski ${ }^{4}$ \\ Colleen Shaw' \\ Hyo Han' \\ Hatem Soliman' \\ Brian Czerniecki' \\ 'Department of Breast Oncology, \\ H. Lee Moffitt Cancer Center and \\ Research Institute, Tampa, FL, USA; \\ 2Morsani College of Medicine, \\ University of South Florida, Tampa, \\ FL, USA; ${ }^{3}$ Moffitt Biomedical Library, \\ H. Lee Moffitt Cancer Center and \\ Research Institute, Tampa, FL, USA; \\ ${ }^{4}$ Department of Preventive Medicine, \\ Northwestern University, Chicago, \\ IL, USA
}

\begin{abstract}
Human epidermal growth factor receptor 2 (HER2)-targeted vaccines are under development, but have so far demonstrated only modest clinical efficacy. Additionally, there has been a lack of adequate safety assessment in large-scale prospective clinical trials. Therefore, we performed a meta-analysis of available clinical trial data to summarize the toxicity profiles of these treatments. Literature search was conducted in February 2018. The trials analyzed had at least one study arm consisting of HER2 vaccine monotherapy. Heterogeneity across studies was analyzed using $I^{2}$ statistics. Data were analyzed using random-effects meta-analysis for absolute risk (AR). Eight trials and 248 patients were included. There was no evidence of heterogeneity between studies for grades $3 / 4$ adverse events (AEs) or for death. The AR for treatment-related serious AEs was $5 \%$ with no treatment-related deaths. The AR of all-grade fatigue, injection site reaction, and fever/chills/rigors was 33\%, 23\%, and 31\%, respectively. Asymptomatic drop in left ventricle ejection fraction was rare $(8 \%)$. HER2 vaccines are well tolerated with increased AR of fatigue, injection site reactions, and fever/chills/rigors.
\end{abstract}

Keywords: HER2, vaccines, immunity, dendritic cells, toxicity

\section{Introduction}

Breast cancer is a heterogeneous disease the classification of which is immunohistochemically defined by the presence of hormonal receptors for estrogen, progesterone, and the expression/amplification status of the human epidermal growth factor receptor 2 (HER2) protein/oncogene. ${ }^{1,2}$ Systemic passive immune treatment directed to the HER2 oncoprotein leads to meaningful improvement in clinical outcomes among the subset of patients with breast tumors harboring HER2 overexpression/amplification. HER2targeted antibodies (ie, trastuzumab and pertuzumab) combined with docetaxel improve the median overall survival (MOS) of patients with HER2-positive (HER2 ${ }^{+}$) metastatic breast cancer (MBC) to 56.5 months; historically, patients treated with chemotherapy alone have an estimated MOS of 20.3 months. ${ }^{3,4}$ Notwithstanding recent advances, patients with HER2 ${ }^{+}$MBC will eventually acquire resistance to HER2-targeted therapies and disease progression will ensue. Furthermore, a subset of patients with HER2 ${ }^{+}$ resectable breast cancer will have disease recurrence despite multimodal treatment. ${ }^{5}$

Next-generation sequencing has led to a better understanding of genomic alterations in HER2 ${ }^{+}$breast cancer. It is remarkable that the vast majority of the aberrations appreciated in these tumors are observed at low frequencies (at $<5 \%$, except for PIK3CA [39\%] and P53 [72\%]). ${ }^{6}$ This advancement has allowed for the design of studies assessing the efficacy of drugs that target specific mechanisms of resistance to 
HER2-targeted therapies. In this setting, the development of immune oncology treatment has gained momentum based on the premise that activation of adaptive cellular immunity can lead to significant anti-tumor activity and clinical benefit. ${ }^{7,8}$ Activation of the adaptive branch of the immune system can be initiated via several mechanisms (ie, immune checkpoint inhibitors, adoptive T-cell transfer, and cytokine infusion). These strategies remain, however, in their initial stages of clinical development for the treatment of HER2 ${ }^{+}$breast cancer patients. It should be noted that thus far, randomized clinical trials have shown only modest activity of targeted therapies in the setting of disease progression after HER2targeted treatments. ${ }^{9}$ Furthermore, in the Phase $\mathrm{Ib} / \mathrm{II}$ trial PANACEA, for example, a monoclonal anti-programmed cell death protein 1 antibody, pembrolizumab, was tested in combination with trastuzumab among 58 female patients with trastuzumab-resistant HER2 ${ }^{+}$MBC (presented at San Antonio Breast Cancer Symposium, 2017). Preliminary results of the Phase II portion of the trial showed an overall response rate of $15 \%$ among patients with programmed death-ligand 1 (PD-L1)-positive tumors; no responses were observed among patients with PD-L1-negative tumors. ${ }^{10}$ The median duration of disease control was 11.1 months, suggesting significant clinical benefit of the treatment combination among a subset of patients with HER2 ${ }^{+}$MBC refractory to HER2-targeted therapies when compared with historical controls treated with ado-trastuzumab emtansine alone. ${ }^{11}$

Active immune therapy with vaccines is an attractive treatment modality as it presents a potentially self-sustained strategy for activation of the immune system, encompassing not only cellular but also humoral components. In addition, the therapy can provoke interactions with the innate immune system, leading to antitumor activity that is not limited to the adaptive immune system, and it may also produce epitope spreading and activation of immune response against other tumor-associated or tumor-specific antigens. ${ }^{12}$ Thus far, HER2 vaccines have shown only modest clinical efficacy among patients with HER2 ${ }^{+}$breast cancer. There is also a lack of large prospective trials assessing the safety of these treatments. Hence, the goal of this systematic review and meta-analysis is to better define the toxicity profile of the HER2-directed vaccines among patients with HER2 ${ }^{+}$ breast cancer.

\section{Methods}

\section{Search strategy}

A systematic literature search was performed in February 2018 by a medical librarian, using MEDLINE via PubMed
(1946-2018), Embase (1947-2018), and the Cochrane Library. All publication dates were included (Tables S1-S4). Selected manuscripts were uploaded to the Covidence online platform at www.covidence.org for the purposes of managing references, identifying and removing duplicates, and reviewing titles and abstracts.

\section{Selection of trials and data extraction}

There were two inclusion criteria for clinical trials. First, they were required to be any-phase trials among patients with $\mathrm{HER}^{+}$breast cancer. HER2 ${ }^{+}$breast cancer is defined by HER2 overexpression in tumor tissue (eg, HER2 overexpression with immunohistochemistry score $3^{+}$) and/or HER2 amplification in tumor tissue (eg, HER2 gene amplification in the primary tumor or metastatic lesion, as determined by fluorescence in situ hybridization [ratio $>2$ and HER 2 copy number $>4$ define HER $2^{+}$disease]). ${ }^{2}$ Second, the trials must have used HER2 vaccines as monotherapy to be included. Pediatric clinical trials and studies assessing the efficacy of HER2 vaccines in combination with other treatments were excluded.

From manuscripts meeting inclusion criteria, we extracted the total number of 1) patients evaluable for toxicity, 2) all-grade AEs, 3) grades 3/4 toxicities, 4) deaths, and 5) patients who discontinued treatment due to toxicities. The number of selected all-grade AEs was also documented (ie, fatigue, nausea, diarrhea, fever, injection site reaction, symptomatic congestive heart failure, and asymptomatic drop in left ventricle ejection fraction [LVEF]). These AEs were selected because they represent the most commonly observed AEs that are associated with HER2-targeted monoclonal antibodies or because of their clinical relevance. The relationship between AEs and treatment administration (ie, treatment-related AEs vs all-causality AEs) was also documented when the information was available. Data extraction was first performed by one primary reviewer and then independently reviewed by one secondary reviewer following PRISMA guidelines.

\section{Statistical methods}

Meta-analyses were conducted using one-sample proportions to obtain the pooled fixed-effect and random-effect estimates. Heterogeneity was assessed via the $I^{2}$ statistic and publication bias was evaluated via the Egger test. Finally, using the Cochran $Q$ test, we also determined betweenstudies heterogeneity caused by differences in subsets of tumor type, line of therapy, treatment, and participant age and performance statuses. All analyses were conducted using R 3.3.3 software package. 


\section{Results}

\section{Included and excluded studies}

Our librarian-guided literature search yielded 1,114 publications obtained through PubMed, EMBASE, and Cochrane. Nine hundred and fifty-two studies were excluded during the title and abstract screening on the basis of their not being prospective randomized trials (Figure 1). After text review, 93 additional studies were excluded for not meeting the inclusion criteria. Eight studies met the inclusion criteria and data from them were extracted (Table 1). These studies included three Phase II trials, and six of the eight studies investigated HER2-directed dendritic cell (DC1) vaccines in breast cancer stages $0-I V$. None of the eight trials reported the median follow-up time in their data presentation, nor did they clearly state the causality of reported AEs.

\section{Description of study participants}

In the eight studies, a total of 248 patients were evaluable for toxicity, 60 of whom had stage IV breast cancer. The median age of study participants was only reported in three studies (50-60 years of age).

\section{Study-to-study heterogeneity and publication bias}

$I^{2}$ statistics revealed that inter-study heterogeneity was $0 \%$ for grades $3 / 4 \operatorname{AEs}(P=0.63)$ and $10 \%$ for serious AEs $(P=0.34)$. There was no significant heterogeneity associated with the outcome of death. Heterogeneity testing for selected grades 3/4 AEs is presented in Table 2. Testing for publication bias did not show significant results.

\section{Number of all-grade AEs, grades 3/4 AEs, serious $A E s$, treatment-related deaths, and number of patients who discontinued treatment due to toxicity}

The total number of all-grade AEs was not meta-analyzed because only a few trials had reported them (two of the eight studies). Eight grades 3/4 AEs were observed among the 248 patients, and there was an estimated pooled absolute risk (AR) of $6 \%$ (95\% CI, 3-10). Four out of eight patients presenting with grade 3 AEs had AEs that were classified as possibly treatment related (ie, injection site reaction, fatigue, diarrhea, and asthenia). There were no reports of grade 4

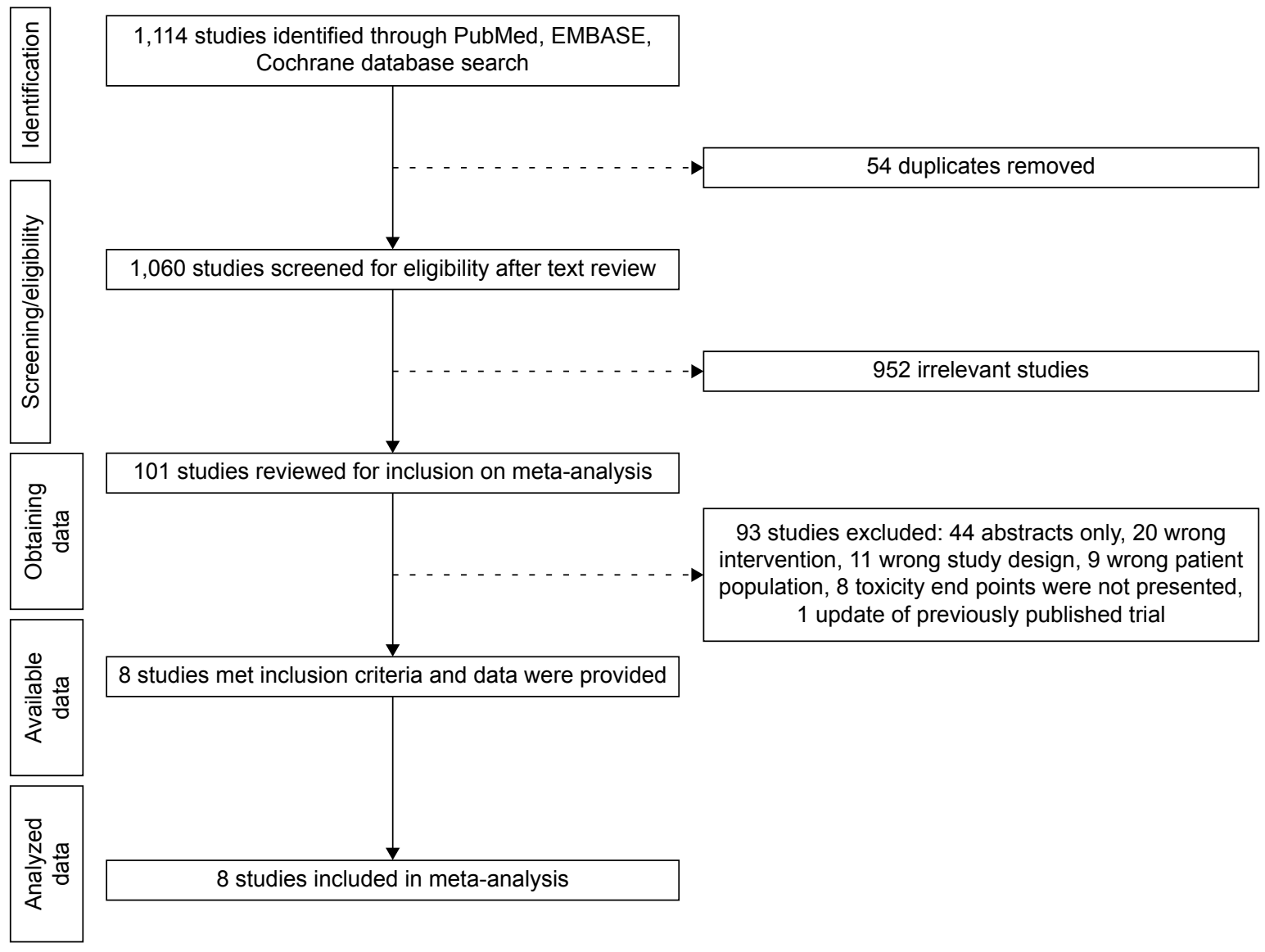

Figure I Study flow diagram. 
Table I Characteristics of clinical trials included in meta-analysis ${ }^{\mathrm{a}}$

\begin{tabular}{|l|l|l|l|l|l|l|l|l|}
\hline PMID & $\begin{array}{l}\text { First } \\
\text { author }\end{array}$ & $\begin{array}{l}\text { Study } \\
\text { phase }\end{array}$ & Stage & $\begin{array}{l}\text { Evaluable } \\
\text { patients, N }\end{array}$ & $\begin{array}{l}\text { Vaccine } \\
\text { type }\end{array}$ & $\begin{array}{l}\text { Vaccine } \\
\text { adjunct }\end{array}$ & $\begin{array}{l}\text { NCI CTCAE } \\
\text { grading }\end{array}$ & $\begin{array}{l}\text { Echo/MUGA performed } \\
\text { during study }\end{array}$ \\
\hline 17293384 & Czerniecki & II & DCIS and microinvasive & I3 & DCs & NA & NR & NR \\
\hline 27965306 & Lowenfeld & II & DCIS and I-III & 54 & DCs & NA & NR & Yes \\
\hline 22252842 & Sharma & I & DCIS & 29 & DCs & NA & NR & Yes \\
\hline 26975189 & Curigliano & II & Stage IV & 40 & dHER2 & ASI5 & NR & Yes \\
\hline I7822557 & Morse & Pilot & Stages I-IV & 6 & DCs & NA & NR & Yes \\
\hline 22130160 & Koski & Pilot & DCIS & 27 & DCs & NA & NR & Yes \\
\hline I77044II6 & Park & I & Stage IV & I8 & DCs & NA & NR & Yes \\
\hline 2699313 I & Limentani & I & Stages I-III & $6 I$ & dHER2 & NA & 2 & Yes \\
\hline
\end{tabular}

Notes: ${ }^{a}$ Number of patients with Eastern Cooperative Oncology Group performance status $\geq 2$ was not reported in any study. ${ }^{\mathrm{b} C a r d i a c}$ toxicity end points for trial published by Koski et al were republished in Bahl et al (PMID: 19800453).

Abbreviations: DCs, dendritic cells; DCIS, ductal carcinoma in situ; dHER2, a truncated recombinant HER2/neu peptide; Echo, echocardiogram; MUGA, multigated acquisition scan; NA, not applicable; NCI CTCAE, National Cancer Institute Common Terminology Criteria Adverse Events; NR, not reported; PMID, PubMed identification.

treatment-related AEs. All eight studies reported the number of patients who discontinued treatment because of toxicities, and none had done so.

Data on serious AEs were available in four of the eight studies, and the random- and fixed-effects pooled AR risk of serious AEs was 5\% (95\% CI, 2-10) (Figure 2). A total of six treatment-related AEs were reported. These were fatigue, malaise, influenza-like symptoms, hypotension, and two episodes of pyrexia. All eight studies reported that there were no treatment-related deaths.

\section{Number of all-grade selected toxicities}

The most common all-grade toxicities were fever, chills, and rigors, with a random-effect absolute risk of $31 \%(95 \% \mathrm{CI}$, $13-56)$, followed by fatigue, with a random-effect AR of $28 \%$ (95\% CI, 12-53) (Table 2). A pooled analysis of injection site reaction showed a pooled AR of $23 \%$ for all-grade reactions. Nonetheless, the fixed-effect AR of an asymptomatic drop in LVEF was $8 \%$ (95\% CI, 6-13). Of note, no symptomatic drop in LVEF was reported.

\section{Discussion}

In light of the growth of immune therapies for the treatment of solid tumors - as exemplified by the approval of numerous checkpoint inhibitors - the development of active immune therapy with vaccines, alone or in combination, has gained renewed momentum. Although vaccine trials for the treatment of HER $2^{+}$breast cancer have hitherto failed to translate clinically meaningful results into daily practice, building a better understanding of the immune milieu may allow for the development of synergistic treatments. For example, in a breast cancer pre-clinical model, blocking the programmed death cell protein/PD-L1 and cytotoxic T-lymphocyte associated protein 4/CD28 molecule pathways with monoclonal antibodies restores antigen-presenting cell function, improves $\mathrm{T}$-cell function (resulting in increased interferon gamma and tumor necrosis factor alpha production), and promotes T-cell proliferation and T-cell targeting of tumors. ${ }^{13}$ Collectively, these data suggest that combined vaccines and checkpoint inhibitors could further activate adaptive immune responses against breast cancer cells.

Table 2 AR of selected all-grade AEs

\begin{tabular}{|c|c|c|c|c|}
\hline All-grade AE & $\begin{array}{l}\text { Fixed-effect AR, } \\
\%(95 \% \mathrm{Cl} \text {, range) }\end{array}$ & $\begin{array}{l}\text { Random-effect AR, } \\
\%(95 \% \mathrm{CI} \text {, range) }\end{array}$ & $I^{2}, \%^{\mathrm{a}}$ & $P$-value \\
\hline Injection site reaction & $31(24-39)$ & $23(9-48)$ & 87 & 0.01 \\
\hline Fever, chills, rigors & $31(23-40)$ & $31(13-56)$ & 83 & 0.01 \\
\hline Fatigue & $33(24-43)$ & $28(I 2-53)$ & 78 & 0.01 \\
\hline Nausea $^{\mathrm{b}}$ & $20(10-36)$ & $13(1-73)$ & 91 & 0.01 \\
\hline Diarrheab $^{\mathrm{b}}$ & $5(2-11)$ & $5(2-11)$ & 0 & 0.88 \\
\hline Dizziness $^{b}$ & $7(3-15)$ & $7(3-15)$ & 0 & 0.35 \\
\hline Asymptomatic drop in LVEF & $8(6-13)$ & $8(6-13)$ & 0 & 0.05 \\
\hline
\end{tabular}

Notes: a Percentage of variance between studies that is due to heterogeneity and not by chance. 'bata were pooled from two studies that reported outcomes. Abbreviations: AEs, adverse events; AR, absolute risk; LVEF, left ventricle ejection fraction. 


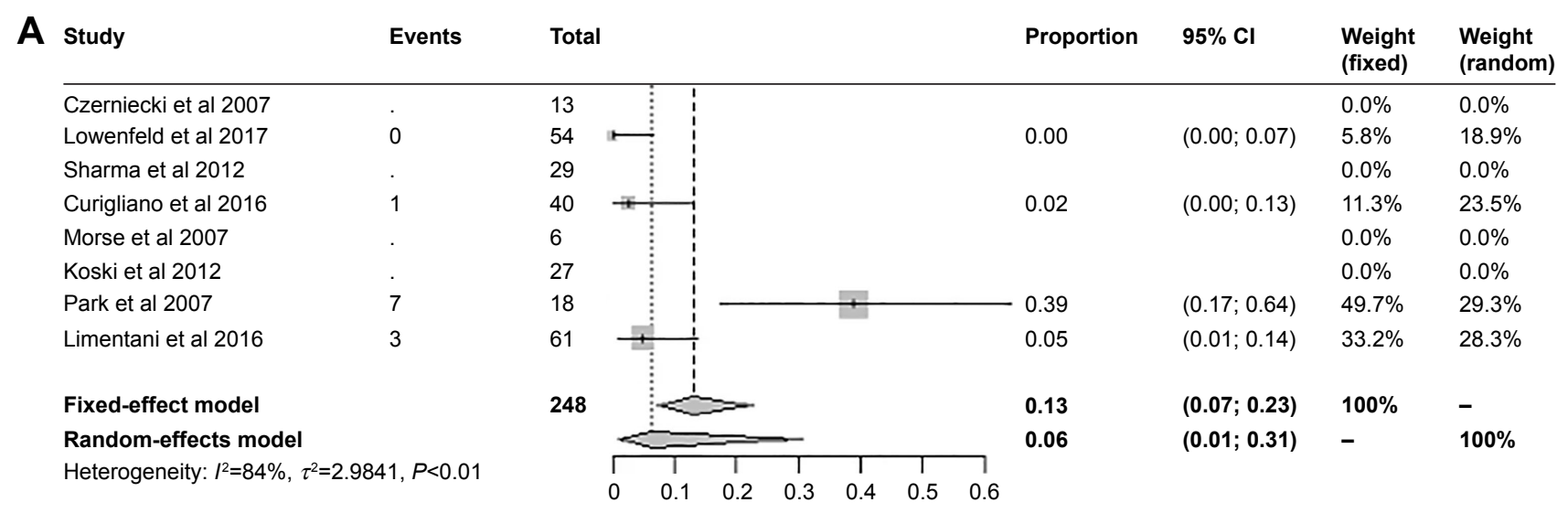

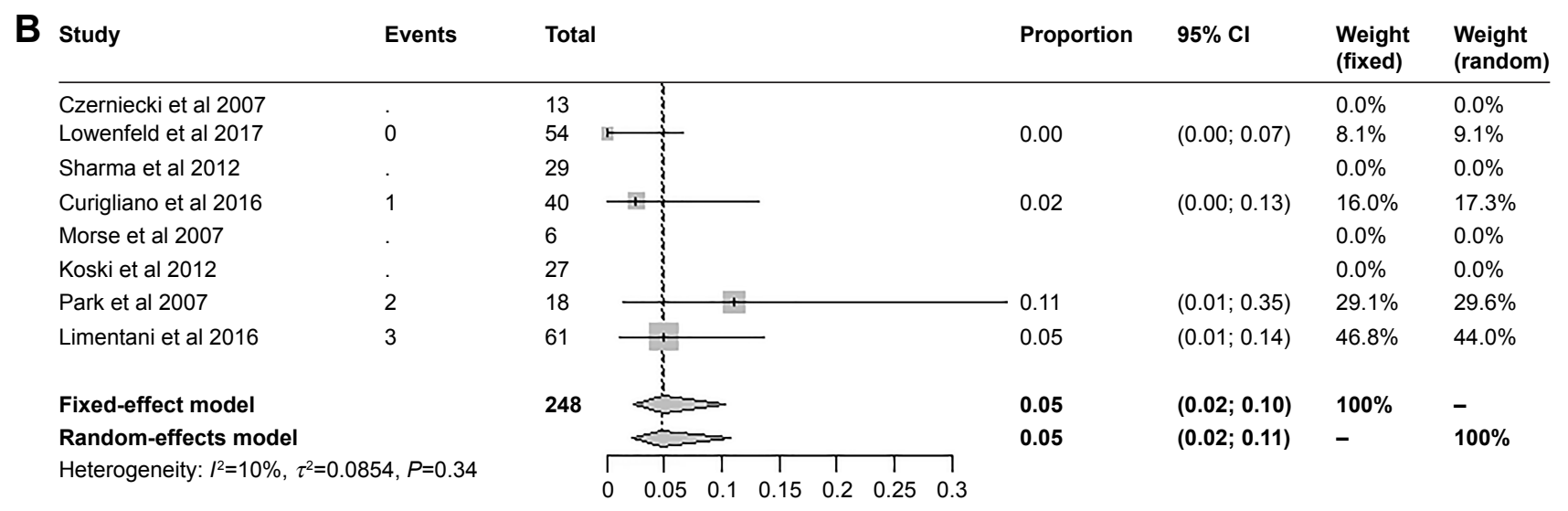

Figure 2 Absolute risk of grade 3/4 AEs (A) and absolute risk of serious AEs (B).

Note: - indicates missing data.

Abbreviation: $\mathrm{AE}$, adverse event.

We conducted a systematic review and meta-analysis to refine the assessment of AE risk associated with HER2 vaccines in patients with HER2 ${ }^{+}$. As a systematic review and meta-analysis of clinical trials, this study improves the precision of AE prevalence estimates among these patients. Eight clinical trials met the inclusion criteria of our study (Table 1). Between-study heterogeneity was observed in studies for some of the end points assessed, which can be explained by differences in baseline performance status, tumor stage, prior number of lines of therapy, and types of vaccine used. Treatment with HER2-directed vaccines was well tolerated. Remarkably, no patient discontinued treatment because of treatment-related toxicities, and only four out of 248 patients had treatment-related grade $3 \mathrm{AEs}$; this was observed among patients with $\mathrm{MBC}$ receiving recombinant HER2 protein and immunostimulant AS15. ${ }^{14}$ These AEs, which included injection site reaction, fatigue, diarrhea, and asthenia, were considered manageable. HER2-targeted therapy with monoclonal antibodies has been associated with an asymptomatic drop in LVEF or New York Heart Association class II congestive heart failure, with an AR of $0.5 \%-11 \%$, which is similar to our findings $(8 \% \mathrm{AR})$ and indicates the favorable cardiac toxicity profile of HER2-directed vaccines. ${ }^{15}$

Lack of access to individual patient data is the major limitation of this study, preventing further exploration of meaningful correlations between patients' characteristics and risks of toxicities. Furthermore, in order to minimize between-study heterogeneity and increase the precision of our results, we did not analyze studies that assessed the efficacy of HER2-vaccines combined with HER2-targeted agents. It should be noted, however, that the reported toxicity profile of HER2 vaccines combined with HER2 monoclonal antibodies does not seem to significantly increase the AR of AEs among patients with HER2 $2^{+}$breast cancer. For example, Disis et al reported the results of a Phase I/II trial in which 22 HLA-A2-positive patients with HER2 ${ }^{+}$MBC, already undergoing treatment with trastuzumab, were treated with major histocompatibility complex classes I and II HER2 epitope vaccines combined with trastuzumab. ${ }^{16}$ The treatment combination was well tolerated and was associated 
with negligible risk (1\%) for grades 3/4 AEs. Additionally, only three treatment-related AEs were reported, including injection-site reaction, ulceration, and fainting.

In summary, treatment with HER2 vaccines is well tolerated and associated with low AR of death and treatment discontinuation caused by treatment-related toxicity. There is increased AR of low-grade infusion site reaction, fever, and fatigue, which do not lead to significant risks of grades 3/4 AEs. Moving forward, the efficacy and safety of HER2 vaccines, such as the dendritic cell vaccine (DC1) and the DNA plasmid-based vaccine (Wokvac), will continue to be developed for the treatment of HER2 ${ }^{+}$breast cancer (NCT03384914, NCT01730118).

\section{Acknowledgments}

We thank Paul Fletcher and Sonya Smyk (Moffitt Cancer Center) for their editorial assistance. They were not compensated beyond their regular salaries. No funding was received for the conduct of this systematic review and meta-analysis.

\section{Disclosure}

B Czerniecki has a patent filed for DC1 vaccine and intellectual property license with ImmunoRestoration. The authors report no other conflicts of interest in this work.

\section{References}

1. Hammond ME, Hayes DF, Dowsett M, et al. American Society of Clinical Oncology/College Of American Pathologists guideline recommendations for immunohistochemical testing of estrogen and progesterone receptors in breast cancer. J Clin Oncol. 2010;28(16):2784-2795.

2. Wolff AC, Hammond ME, Hicks DG, et al. Recommendations for human epidermal growth factor receptor 2 testing in breast cancer: American Society of Clinical Oncology/College of American Pathologists clinical practice guideline update. J Clin Oncol. 2013;31(31):3997-4013.

3. Slamon DJ, Leyland-Jones B, Shak S, et al. Use of chemotherapy plus a monoclonal antibody against HER2 for metastatic breast cancer that overexpresses HER2. N Engl J Med. 2001;344(11):783-792.
4. Swain SM, Baselga J, Kim SB, et al. Pertuzumab, trastuzumab, and docetaxel in HER2-positive metastatic breast cancer. $N$ Engl J Med. 2015;372(8):724-734.

5. de Azambuja E, Holmes AP, Piccart-Gebhart M, et al. Lapatinib with trastuzumab for HER2-positive early breast cancer (NeoALTTO): survival outcomes of a randomised, open-label, multicentre, phase 3 trial and their association with pathological complete response. Lancet Oncol. 2014;15(10):1137-1146.

6. Cancer Genome Atlas Network. Comprehensive molecular portraits of human breast tumours. Nature. 2012;490(7418):61-70.

7. Borghaei H, Paz-Ares L, Horn L, et al. Nivolumab versus docetaxel in advanced nonsquamous non-small-cell lung cancer. $N$ Engl J Med. 2015;373(17):1627-1639.

8. Reck M, Rodríguez-Abreu D, Robinson AG, et al. Pembrolizumab versus chemotherapy for PD-L1-positive non-small-cell lung cancer. N Engl J Med. 2016;375(19):1823-1833.

9. André F, O’Regan R, Ozguroglu M, et al. Everolimus for women with trastuzumab-resistant, HER2-positive, advanced breast cancer (BOLERO-3): a randomised, double-blind, placebo-controlled phase 3 trial. Lancet Oncol. 2014;15(6):580-591.

10. Loi S, Giobbe-Hurder A, Bachelot T. Phase $1 \mathrm{~b} / 2$ study evaluating safety and efficacy of pembrolizumab and trastuzumab in patients with trastuzumab-resistant HER2-positive metastatic breast cancer: results from the PANACEA (IBCSG 45-13/BIG 4-13/KEYNOTE-014) study. San Antonio Breast Cancer Symposium; December 5-9; 2017; San Antonio, TX.

11. Verma S, Miles D, Gianni L, et al. Trastuzumab emtansine for HER2positive advanced breast cancer. NEngl JMed.2012;367(19):1783-1791.

12. Costa RLB, Soliman H, Czerniecki BJ. The clinical development of vaccines for HER2 ${ }^{+}$breast cancer: Current landscape and future perspectives. Cancer Treat Rev. 2017;61:107-115.

13. Ge Y, Xi H, Ju S, Zhang X. Blockade of PD-1/PD-L1 immune checkpoint during $\mathrm{DC}$ vaccination induces potent protective immunity against breast cancer in hu-SCID mice. Cancer Lett. 2013;336(2):253-259.

14. Curigliano G, Romieu G, Campone M, et al. A phase I/II trial of the safety and clinical activity of a HER2-protein based immunotherapeutic for treating women with HER2-positive metastatic breast cancer. Breast Cancer Res Treat. 2016;156(2):301-310.

15. Costa RB, Kurra G, Greenberg L, Geyer CE. Efficacy and cardiac safety of adjuvant trastuzumab-based chemotherapy regimens for HER2positive early breast cancer. Ann Oncol. 2010;21(11):2153-2160.

16. Disis ML, Wallace DR, Gooley TA, et al. Concurrent trastuzumab and HER2/neu-specific vaccination in patients with metastatic breast cancer. J Clin Oncol. 2009;27(28):4685-4692. 


\section{Supplementary materials}

Table SI Keywords used in systematic review by category

\begin{tabular}{|c|c|c|c|c|}
\hline HER2 keywords & $\begin{array}{l}\text { HER2 vaccine } \\
\text { keywords }\end{array}$ & $\begin{array}{l}\text { Breast cancer } \\
\text { keywords }\end{array}$ & Toxicity keywords & Clinical trials keywords ${ }^{a}$ \\
\hline $\begin{array}{l}\text { dHER2 } \\
\text { HER2 } \\
\text { HER2/neu } \\
\text { "Receptor, ErbB-2"[Mesh] } \\
\text { human epidermal growth } \\
\text { factor receptor } 2 \\
2 \text { her } \\
\text { erbb2 } \\
\text { neu Gene } \\
\text { NEU proto-oncogene } \\
\text { neu receptor } \\
\text { TKRI }\end{array}$ & $\begin{array}{l}\text { Her2 Vaccine } \\
\text { Cancer Vaccines[Mesh] } \\
\text { Cancer Vaccines } \\
\text { NeuVax } \\
\text { Peptide cancer vaccines } \\
\text { peptide vaccines } \\
\text { Nelipepimut-S } \\
\text { GP2 } \\
\text { AE37 } \\
\text { "E75" } \\
\text { E75 HER2-derived peptide } \\
\text { vaccine, nelipepimut-S } \\
\text { whole cell vaccine } \\
\text { CHP-HER2 vaccine } \\
\text { [Supplementary Concept] }\end{array}$ & $\begin{array}{l}\text { breast cancer } \\
\text { breast neoplasms }\end{array}$ & $\begin{array}{l}\text { Receptor, ErbB-2/toxicity[Mesh] } \\
\text { Cancer Vaccines/adverse } \\
\text { effects[Mesh] } \\
\text { Cancer Vaccines/administration } \\
\text { and dosage[Mesh] } \\
\text { Toxicity } \\
\text { toxicity [Subheading] }\end{array}$ & $\begin{array}{l}\text { (clinical trials OR “clinical trial" } \\
\text { OR Clinical Trial[ptyp] OR “phase } \\
\text { I" OR "phase Il” OR “phase 2" } \\
\text { OR "phase I" OR "phase I/2" } \\
\text { OR "Phase I/Il" OR randomized } \\
\text { controlled trial[pt] OR } \\
\text { controlled clinical trial[pt] } \\
\text { OR randomized[tiab] } \\
\text { OR randomised[tiab] OR } \\
\text { randomization[tiab] OR } \\
\text { randomisation[tiab] OR } \\
\text { placebo[tiab] OR drug therapy[sh] } \\
\text { OR randomly[tiab] OR trial[tiab] } \\
\text { OR groups[tiab]) NOT } \\
\text { (animals[mh] NOT humans[mh]) } \\
\text { NOT (Editorial[pt] OR Letter[pt] } \\
\text { OR Case Reports[pt] OR } \\
\text { Comment[pt]) }\end{array}$ \\
\hline
\end{tabular}

Note: aBased on the RCT filter from Cochrane.

Abbreviation: RCT, randomized controlled trial.

Table S2 Medical subject headings (MeSH) terms search and synonyms

\begin{tabular}{|c|c|c|}
\hline Terms & Search results ${ }^{a}$ & Database $^{b}$ \\
\hline \multicolumn{3}{|l|}{ Synonyms } \\
\hline HER2 vaccine & 5 & 5 \\
\hline Vaccine & 74 & 7,677 \\
\hline Vaccination & 30 & $4,|9|$ \\
\hline Inoculations & 6 & 141 \\
\hline Immunization & 5 & 1,492 \\
\hline VACCIN & - & 28 \\
\hline HER2 & 74 & 1,956 \\
\hline human epidermal growth factor receptor 2 & 9 & 294 \\
\hline 2 her & 2 & 5 \\
\hline erbb2 & 2 & 168 \\
\hline neu Gene & - & 3 \\
\hline NEU proto-oncogene & - & 1 \\
\hline neu receptor & - & 10 \\
\hline TKRI & - & 2 \\
\hline
\end{tabular}

Notes: -, No studies found. ${ }^{2}$ Number of studies in the search results containing the term or synonym. ${ }^{b}$ Number of studies in the entire database containing the term or synonym. 
Table S3 PubMed and Embase search strategy and results

\begin{tabular}{|l|l|l|}
\hline Number & Query & Results $^{\mathbf{a}}$ \\
\hline 1 & her2 & 40,244 \\
\hline 2 & erbb2 & 9,929 \\
\hline 3 & "neu gene" & 887 \\
\hline 4 & "epidermal growth factor receptor 2" & 43,228 \\
\hline 5 & \#I OR \#2 OR \#3 OR \#4 & 66,477 \\
\hline 6 & "her2 vaccine" & 54 \\
\hline 7 & "cancer vaccine" & 14,660 \\
\hline 8 & "nelipepimut s" & 100 \\
\hline 9 & "peptide vaccine" & 4,070 \\
\hline 10 & \#6 OR \#7 OR \#8 OR \#9 & 17,673 \\
\hline 11 & \#5 AND \#I0 & 940 \\
\hline 12 & \#II AND [embase]/lim NOT ([embase]/ & 331 \\
\hline & lim AND [medline]/lim) & \\
\hline
\end{tabular}

Notes: aNumber of studies in the database containing the query term. PubMed Search String (PubMed.gov): ((dHER2 OR HER2 OR HER2/neu OR "Receptor, ErbB-2"[Mesh] OR human epidermal growth factor receptor 2 OR 2 her OR erbb2 OR neu Gene OR NEU proto-oncogene OR neu receptor OR TKRI)) AND (Her2 Vaccine OR Cancer Vaccines[Mesh] OR Cancer Vaccines OR NeuVax OR Peptide cancer vaccines OR peptide vaccines OR Nelipepimut-S OR GP2 OR AE37 OR "E75" OR E75 HER2-derived peptide vaccine, nelipepimut-S OR whole cell vaccine OR CHP-HER2 vaccine [Supplementary Concept]). Embase Search String (Embase.com): ((her2 OR erbb2 OR "neu gene" OR "epidermal growth factor receptor 2") AND ("her2 vaccine" OR "cancer vaccine" OR "nelipepimuts" OR "peptide vaccine")) AND [embase]/lim NOT ([embase]/ $\lim$ AND [medline]/lim).

Table S4 Cochrane search strategy and results (Cochrane search string)

\begin{tabular}{|l|l|l|}
\hline Number & Query & Results $^{\mathbf{a}}$ \\
\hline $\mathrm{I}$ & HER2 & 2,933 \\
\hline 2 & $\begin{array}{l}\text { MeSH descriptor: [Cancer Vaccines] } \\
\text { explode all trees }\end{array}$ & 294 \\
\hline 3 & \#I and \#2 & 6 \\
\hline
\end{tabular}

Note: ${ }^{a}$ Obtained on February 8, 2018.

\section{Publish your work in this journal}

Drug Design, Development and Therapy is an international, peerreviewed open-access journal that spans the spectrum of drug design and development through to clinical applications. Clinical outcomes, patient safety, and programs for the development and effective, safe, and sustained use of medicines are the features of the journal, which has also been accepted for indexing on PubMed Central. The manuscript management system is completely online and includes a very quick and fair peer-review system, which is all easy to use. Visit $\mathrm{http}: / /$ www.dovepress.com/testimonials.php to read real quotes from published authors.

Submit your manuscript here: http://www.dovepress.com/drug-design-development-and-therapy-journal 